\title{
Comunicación en tiempos de COVID-19, uso efectivo de las señales visuales militares modificadas en momentos quirúrgicos, solución a un problema
}

\author{
Communication in times of COVID-19, effective use of modified military \\ visual signals in surgical moments, solution to a problem
}

\author{
Nicolás Daniel Sánchez-Hernández ${ }^{1} \mathbb{D}$, Lu is Felipe Cabrera-Vargas² $\mathbb{D}$, \\ Ricardo Adolfo Villarreal-Viana ${ }^{3}$ (D)
}

1. Médico General, Universidad El Bosque, Bogotá D.C., Colombia.

2. Médico especialista en Cirugía General, Fundación Santa Fe de Bogotá; profesor de cirugía, Universidad de los Andes y Universidad El Bosque; miembro Comité de Comunicaciones Asociación Colombiana de Cirugía. Bogotá D.C., Colombia.

3. Médico especialista en Cirugía General y Gastrointestinal, Los Cobos Medical Center; profesor de Cirugía, Universidad El Bosque, Bogotá D.C., Colombia.

\section{Resumen}

La pandemia por el nuevo coronavirus SARS-CoV-2 ha causado gran impacto tanto en los diferentes espacios hospitalarios como en los no hospitalarios, ocasionando gran número de muertes dada su gran capacidad de diseminación. Esta situación nos ha llevado a implementar constantes estrategias de cambio con el fin de proteger la integridad del personal médico y de los pacientes.

Es por esto que, guiados por las grandes asociaciones como la World Health Organization, el American College of Surgeons, los Centers for Disease Control and Prevention y la Asociación Colombiana de Cirugía, se ha planteado una serie de protocolos para el buen uso de los elementos de protección personal, que en los diferentes momentos quirúrgicos hacen difícil la comunicación asertiva. Por lo anteriormente expuesto, se quiere presentar el uso de las señales visuales militares modificadas como la solución para un problema en los procedimientos quirúrgicos en los tiempos de la COVID.

Palabras clave: COVID-I9; infecciones por coronavirus; pandemias; cirugía general; quirófanos; lengua de signos.

Fecha de recibido: 29/05/2020 - Fecha de aceptación: 31/05/2020

Correspondencia: luis.felipe.cabrera@hotmail.com

Citar como: Sánchez-Hernández ND, Cabrera-Vargas LF, Villarreal-Viana RA. Comunicación en tiempos de COVID-19, uso efectivo de las señales visuales militares modificadas en momentos quirúrgicos, solución a un problema. Rev Colomb Cir. 2020;35:373-7. https://doi.org/10.30944/20117582.681

Este es un artículo de acceso abierto bajo una Licencia Creative Commons - BY-NC-ND https://creativecommons.org/licenses/by-ncnd/4.0/deed.es 


\begin{abstract}
The pandemic due to the new SARS-CoV-2 has caused a great impact both in the different hospital and non-hospital spaces, causing a large number of deaths due to its great capacity for dissemination. This situation has led us to implement constant change strategies in order to protect the integrity of medical personnel and patients. That is why, guided by large associations such as the World Health Organization, the American College of Surgeons, the Centers for Disease Control and Prevention, and the Asociación Colombiana de Cirugía, a series of protocols have been proposed for the proper use of elements of personal protection, which at different surgical times makes assertive communication difficult. For the above, we want to present the use of modified military visual signs as the solution to a problem in surgical procedures in times of COVID-I9.
\end{abstract}

Key words: COVID-I9; coronavirus infections; pandemics; general surgery; operating rooms; sign language.

\section{Introducción}

La pandemia por SARS-CoV-2 ha llevado a la medicina a constantes cambios en los últimos meses; asimismo, en la práctica clínica ha obligado al personal médico a ser más exhaustivo con los protocolos de asepsia y al uso de los elementos de protección personal.

En el ámbito quirúrgico, los cirujanos se han visto obligados a posponer los procedimientos quirúrgicos no vitales y a acortar la ocupación de las salas de cirugía, con el fin de minimizar el riesgo de infección ${ }^{1}$. Las entidades quirúrgicas de urgencia no se deben posponer ni se debe demorar su atención en la situación de pandemia, entre ellas, cabe mencionar la apendicitis, la peritonitis, las cesáreas y las cirugías por trauma ${ }^{2}$.

Para la atención segura tanto del equipo quirúrgico como del paciente, se deben tener en cuenta varios aspectos, tales como la COVID-I9 del paciente y el contar con suficientes elementos de protección personal para el equipo quirúrgico. En el momento de la intubación, solamente debe estar presente el personal imprescindible ${ }^{2,3}$.

Se ha encontrado que el uso de los elementos de protección personal en los diferentes procedimientos quirúrgicos durante la pandemia, dificulta la correcta comunicación del equipo quirúrgico (cirujano, anestesiólogo, ayudante quirúrgico, instrumentadora quirúrgica). Por este motivo, se presenta la experiencia de la comunicación mediante señales militares visuales modificadas en una institución de cuarto nivel, como la solución a este problema.

\section{Discusión}

La comunicación asertiva en las salas de cirugía juega un papel importante en factores como el tiempo quirúrgico y los resultados para el paciente. Las señales visuales son un modo de comunicación que se puede usar para transmitir, rápidamente y a distancias cortas ${ }^{4}$, mensajes previamente organizados, por lo cual su uso es común en la actualidad en las diferentes fuerzas militares del mundo.

Teniendo en cuenta el índice de mortalidad de las personas asociadas a la atención en salud en otros países y los casos reportados en las últimas semanas en Colombia, son fundamentales los elementos de protección personal en todos los servicios de salud.

En las salas de cirugía se han aumentado los protocolos de seguridad para disminuir el potencial riesgo de contagio por COVID-I9 para el paciente, el personal de las salas de hospitalización y recuperación, y el grupo quirúrgico ${ }^{5,6} \mathrm{El}$ personal de salud que tiene contacto directo con el paciente en procedimientos que no generan aerosoles, debe portar mascarilla quirúrgica, visor, careta o monogafas, bata antifluidos de manga larga, guantes no estériles, vestido quirúrgico debajo de la bata que se retira al finalizar el turno y gorro; el personal que tiene contacto directo con el paciente en procedimientos que generan aerosoles y en los procedimientos quirúrgicos, deben portar una mascarilla N95, visor, careta o monogafas, bata antifluidos de manga larga, guantes no estériles, vestido quirúrgico debajo 
de la bata que se retira al final del turno, gorro y polainas ${ }^{6}$ (figuras I y 2 ).

Durante los diferentes procedimientos quirúrgicos practicados en la temporada de la pandemia, se ha podido evidenciar que los elementos de protección personal -especialmente, la mascarilla N95 y la careta de seguridad-dificultan la comunicación asertiva del equipo quirúrgico, lo cual tiene como resultado retardo en el procedimiento, ineficacia y tensión ${ }^{(4,7,8)}$ (tabla I).

En este momento de la pandemia, se propone utilizar algunas de las señales militares visuales ${ }^{4}$, adaptadas y modificadas para los momentos quirúrgicos actuales (tabla 2).

Con la implementación de estas señales militares básicas, modificadas y adaptadas para nuestra necesidad, se evidenció mayor capacidad de comunicación asertiva no verbal, menos episodios de reiterar órdenes -lo que disminuye el tiempo quirúrgico en 5 a 6 minutos, en comparación con un procedimiento normal-, mayor eficacia y menor tensión en el equipo quirúrgico.

\section{Conclusión}

Actualmente, los cirujanos en todo el mundo están obligados a ser muy acuciosos en el uso de los

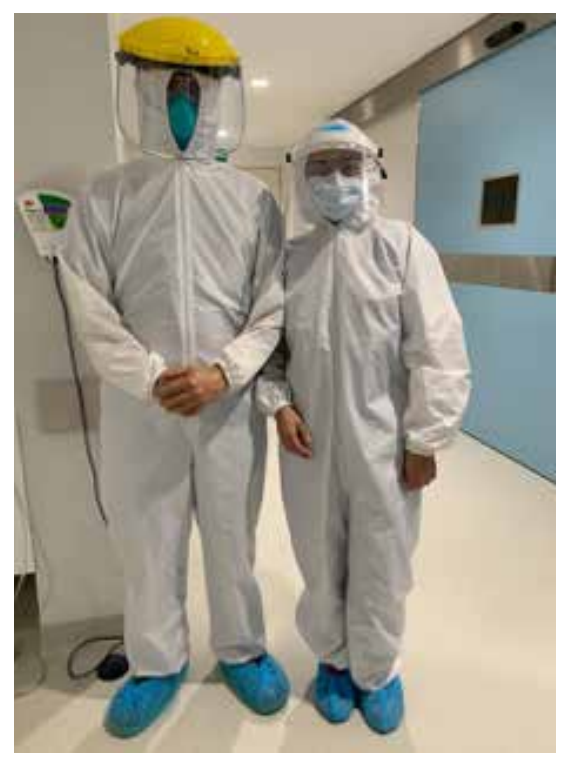

Figura 1. Equipo quirúrgico con elementos de protección personal antes del procedimiento quirúrgico

elementos de protección personal, con el objetivo principal de evitar alguna fuente de contagio por el virus SARS-CoV-2. Estos cambios llevaron a
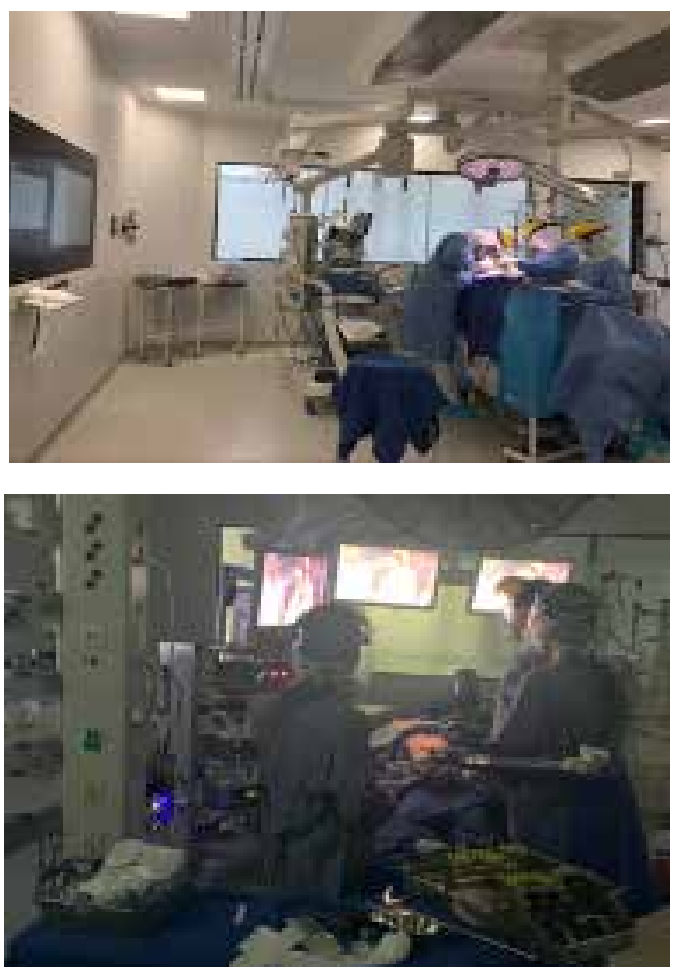

Figura 2. Procedimientos quirúrgicos realizados con el uso de los elementos de protección personal

Tabla 1. Dificultades actuales en la comunicación en quirófano.

\begin{tabular}{ll}
\hline \multicolumn{1}{c}{ Efecto y definición } & \multicolumn{1}{c}{ Ejemplo ilustrativo } \\
\hline Retraso & Se presentaron situaciones \\
$\begin{array}{l}\text { La difícil comunicación gene- } \\
\text { ra minutos de retraso en el } \\
\text { procedimiento. }\end{array}$ & $\begin{array}{l}\text { licitó una pinza específica } \\
\text { (Kelly-Adson, armónica) y, } \\
\text { al no ser clara la petición del } \\
\text { cirujano por los equipos de } \\
\text { protección personal, la ins- } \\
\text { trumentadora no ejecutó la }\end{array}$ \\
& acción.
\end{tabular}

Ineficacia y tensión

El cirujano debe repetir su instrucción o petición; la falta de comunicación genera tensión en los momentos de la cirugía, porque el cirujano debe repetir la solicitud en múltiples ocasiones.
Estos eventos hacen que el cirujano deba reiterar su instrucción en varias ocasiones lo cual genera momentos de tensión. 
Tabla 2. Señas visuales militares adaptadas al tiempo quirúrgico actual de pandemia por SARS-COV-2/ COVID-19.

\begin{tabular}{|l|l|}
\hline Correcto & \\
\hline Entendido & \\
\hline Incorrecto & \\
\hline Seguir & \\
\hline
\end{tabular}

que la comunicación en las salas de cirugía sea deficiente, lo cual aumenta la permanencia en las salas de cirugía y los tiempos quirúrgicos, y pone en riesgo la seguridad del paciente en la eventualidad de una orden no cumplida correctamente. Desde nuestra perspectiva, es útil y viable usar las señales anteriormente descritas, las cuales quedan disponibles para ser adaptadas y modificadas según la necesidad del profesional que las ponga en ejecución.

\section{Cumplimiento de normas éticas}

Consentimiento informado. Este estudio incluye una revisión de la literatura y presenta la experiencia del equipo quirúrgico de una clínica, como tal, no hay necesidad de un consentimiento informado firmado por los pacientes. El Comité de Ética Institucional aprobó el diseño y la metodología del estudio.

Conflicto de interés. Los autores manifiestan que no tienen conflicto de ninguna naturaleza para el desarrollo de la investigación

Fuentes de financiación. Los recursos de financiación del proyecto provienen en su totalidad de aportes de los autores del proyecto de investigación.

\section{Referencias}

I. De Simone B, Chouillard E, Di Saverio S, Pagani L, Sartelli M, Biffl WL, et al. Emergency surgery during the COVID-I9 pandemic: what you need to know for practice. Ann R Coll Surg Engl. 2020;102:323-32. https://doi.org/IO.I308/rcsann.2020.0097

2. Forrester JD, Nassar AK, Maggio PM, Hawn MT. Precautions for Operating Room Team Members During the COVID-I9 Pandemic. J Am Coll Surg. 2020;230:IO98-IIOI. https://doi.org/IO.IoI6/j.jamcollsurg.2020.03.030

3. World Health Organization. Rational use of personal protective equipment for coronavirus disease (COVID-I9): interim guidance, 27 February 2020. World Health Organization. Fecha de consulta: 28 de mayo de 2020. Disponible en:

https://apps.who.int/iris/handle/I0665/3312I5

4. Headquarters, Department of the Army. Visual signals. Washington, DC. September 1987. Fecha de consulta: 28 de mayo de 2020. Disponible en: https://www.rad- 
ford.edu/content/dam/colleges/chbs/rotc/Forms/fm/ Visual\%2oSignals\%2oFM\%202I-6o.pdf.

5. Correia MI, Ramos RF, Von Bahten LC. The surgeons and the COVID-I9 pandemic. Rev. Col. Bras. Cir. 2020; 47: e20202536. Fecha de consulta: 28 de mayo de 2020. https://doi.org/IO.I590/oI0o-699Ie-20202536

6. World Health Organization. Advice on the use of masks in the context of COVID-I9: interim guidance, 6 April 2020. World Health Organization. Fecha de consulta: 28 de mayo de 2020. Disponible en:

https://apps.who.int/iris/handle/I0665/331693
7. Rubio-Pérez I, Badia J, Mora-Rillo M, Martín Quirós A, García Rodríguez J, Balibrea J, et al. COVID-I9: conceptos clave para el cirujano. Cirugía Española. 2020;98:310I9. https://doi.org/IO.IOI6/j.ciresp.2020.04.009

8. Sánchez C, Rodríguez S, Cruz D, Domínguez LC, Vega V, Hoyos C, Duque JC, Gómez C. Caracterización de los patrones de comunicación en salas de cirugía, durante procedimientos en un hospital de tercer nivel. Rev Colomb Cir. 2013;28:3I-8. 